Full Length Article

\title{
Exploring the effects of mental and muscular fatigue in soccer players' performance
}

\author{
Diogo Coutinho $^{\mathrm{a}, *}$, Bruno Gonçalves ${ }^{\mathrm{a}}$, Del P. Wong ${ }^{\mathrm{b}}$, Bruno Travassos ${ }^{\mathrm{c}}$, \\ Aaron J. Coutts ${ }^{\mathrm{d}}$, Jaime Sampaio ${ }^{\mathrm{a}}$ \\ ${ }^{a}$ Research Center in Sports Sciences, Health Sciences and Human Development (CIDESD), CreativeLab Research Community, University of Trás-os- \\ Montes and Alto Douro, Vila Real, Portugal \\ ${ }^{\mathbf{b}}$ Sport Science Research Center, Shandong Sport University, Jinan, China \\ ${ }^{\mathrm{c}}$ Research Center in Sports Sciences, Health Sciences and Human Development (CIDESD), CreativeLab Research Community, Department of Sports \\ Sciences, University of Beira Interior, Portugal \\ ${ }^{\mathrm{d}}$ Human Performance Research Centre, University of Technology Sydney (UTS), Sydney, Australia
}

\section{A R T I C L E I N F O}

\section{Keywords:}

Small-sided games

Time-motion analysis

Fatigue

Team sports

Tactical behavior

\begin{abstract}
A B S T R A C T
This study examined the effects of induced mental and muscular fatigue on soccer players' physical activity profile and collective behavior during small-sided games (SSG). Ten youth soccer players performed a 5vs5 SSG under three conditions: a) control, playing without any previous activity; b) muscular fatigue, playing after performing a repeated change-of-direction task; c) mental fatigue, playing after completing a 30 min Stroop color-word task. Players' positional data was used to compute time-motion and tactical-related variables. The muscular fatigue condition resulted in lower distances covered in high speeds $(\sim 27 \%, 0.3 ; \pm 0.5)$ than the control condition. From the tactical perspective, the muscular fatigue condition resulted in lower distance between dyads and players spent $\sim 7 \%$ more time synchronized in longitudinal displacements than the control condition $(0.3 ; \pm 0.3)$. Additionally, players spent $\sim 14 \%$ more time synchronized with muscular fatigue than with mental fatigue $(0.7 ; \pm 0.3)$. The mental fatigue condition resulted in a very likely more predictable pattern in the distance between dyads than in muscular fatigue condition $(0.4 ; \pm 0.2)$. Also, the mental fatigue possibly decreased the teams' stretch index when compared with control $(0.2 ; \pm 0.3)$ and likely increased compared with muscular fatigue $(0.5 ; \pm 0.5)$. The better levels of longitudinal synchronization after muscular fatigue, might suggest the usage of tactical-related tasks after intense exercise bouts. The lower physical performance and time spent longitudinally synchronized after mental fatigue, should alert to consider this variable before matches or training activities that aim to improve collective behavior.
\end{abstract}

\section{Introduction}

In team sports such as soccer, player's movement behavior (physical and tactical) are shaped by the interaction between the performer, the task and the surrounding environmental constraints (Newell, 1986). Accordingly, performance in many team sports is dependent on the players' ability to identify the appropriate environmental cues that provide opportunities for actions (Travassos,

\footnotetext{
* Corresponding author at: Research Center in Sports Sciences, Health Sciences and Human Development, CIDESD, CreativeLab Research Community, Quinta de Prados, Ap. 202, 5000-911 Vila Real, Portugal.

E-mail address: damcoutinho@utad.pt (D. Coutinho).
} 
Gonçalves, Marcelino, Monteiro, \& Sampaio, 2014). However this performance may be affected by individual factors (Newell, 1986) such as player fatigue. Fatigue has been described as a reduction in the maximal voluntary muscle force, that results from exercise (Gandevia, 2001). From this perspective, there are two possible explanations that lead to this decrease: i) muscle fatigue, when the impairments in the peak force are related to the processes in the muscle cells that affect the muscle contractile functions (Bishop, 2012; Knicker, Renshaw, Oldham, \& Cairns, 2011); or ii) central fatigue, when the decrease in the muscle force output were related with the reduced neural drive from the motor cortex to the motor units (Knicker et al., 2011; Mehta \& Agnew, 2012). Based on these considerations, it is possible that physical and tactical behavior of players may be affected during periods of muscular and/or central fatigue.

It has been shown that changes in the players physical performance during soccer matches (Rampinini et al., 2011), which can be linked not only with contextual events such as team strategy or playing positions (Castellano, Blanco-Villaseñor, \& Alvarez, 2011), but also as result of the muscular fatigue (Bishop, 2012; Nedelec et al., 2012; Tomazin, Morin, \& Millet, 2016). In fact, the demands of soccer matches (Bush, Barnes, Archer, Hogg, \& Bradley, 2015), particularly in high intensity actions and accelerations, results in players experiencing transient muscular fatigue (Silva et al., 2017), which affects their activity profile. For example, it was found impairments in running performance occurred following high-intensity intermittent periods during soccer matches (Varley, Elias, \& Aughey, 2012). The observed drops in distance covered might reach $\sim 50 \%$ less than during the peak period (Bradley et al., 2009). Accordingly, these decreases in the players' activity can be explained by changes in perception of opportunities for action and consequently with changes on tactical movement behavior of players to perform as a team.

The effects of muscular fatigue in team tactical behavior can be demonstrated through changes in positioning and decisionmaking (Sampaio, Gonçalves, Rentero, Abrantes, \& Leite, 2014; Smits, Pepping, \& Hettinga, 2014). For example, Sampaio, Gonçalves, et al. (2014), used the Yo-Yo intermittent recovery level 2 as additional fatiguing task, to analyze how the increased muscular fatigue impacted the players movement behavior during a simulated basketball game. The results showed a higher regularity in players positioning at faster speeds after the additional fatigue task. Higher regularity in players positioning was reported during the progression of each half and was reported to be attributed to accumulated muscular fatigue (Duarte, Araujo, Folgado, et al., 2013). In fact, the increase of players positioning regularity towards the end of soccer matches have been linked earlier with muscular fatigue (Duarte, Araujo, Correia, et al., 2013; Duarte, Araujo, Folgado et al., 2013). While these studies add important findings, they did not have the aim of assess the impact of muscular fatigue on the players positioning, but rather understand how the collective behavior dynamics evolve across a competitive match. Additionally, these studies did not induce fatigue prior to the game, or directly assess the presence of fatigue. Therefore, at present, it remains unclear how muscular fatigue may constraint players movement behavior in soccer. Considering that soccer players are likely to experience periods of transient muscular fatigue (Bradley et al., 2009; Silva et al., 2017; Varley et al., 2012), a better understanding on how players movement behavior changes might provide useful information to the coaches to prepare the players to deal with these scenarios.

From the individual constraints perspective, the mental fatigue may also affect players' behavior. Mental fatigue has been described as psychobiological stated characterized by acute increases in the subjective ratings of mental fatigue and mental effort and cognitive impairments in the ability to maintain attentional focus (Shou \& Ding, 2013), identify and use visual cues (Boksem, Meijman, \& Lorist, 2006), and assess and adjust actions (Lorist, Boksem, \& Ridderinkhof, 2005), which results from experiencing long periods of demanding cognitive activity (Smith, Marcora, \& Coutts, 2015; Van Cutsem et al., 2017). Accordingly, previous studies on soccer players have shown that mental fatigue can reduce the technical performance, as well as the physical performance, mainly at low intensities (Badin, Smith, Conte, \& Coutts, 2016; Smith, Coutts, et al., 2016; Smith, Zeuwts, et al., 2016). Indeed laboratory studies showed that the mental fatigue - induced through a 30-min Stroop color-word task - increased perception of effort during physical activity and reduced soccer-specific running capacity, short passing and shooting performance (Smith, Coutts, et al., 2016), and also reduced the speed and accuracy of soccer-specific decision-making tasks (Smith, Zeuwts, et al., 2016).

The majority of studies have used laboratory environments to explore the effects of mental fatigue in soccer (Smith, Coutts, et al., 2016; Smith, Zeuwts, et al., 2016). However, given the complex and unique nature of soccer, different results may emerge when analyzing the effects of mental fatigue under more practical approaches. In this regard, a field study showed that mental fatigue impaired technical but not physical performance during soccer game-based situations in youth players (Badin et al., 2016). More recently, Coutinho et al. (2017) used a motor coordination task to induce mental fatigue and analyzed how players physical and movement behavior was affected by the mental fatigue. While no effects were found in the physical variables, the team movement behavior results showed decreases in movement synchronization and in positional adjustments such as speed of contraction, when mentally fatigued. However, in these two field studies only one team was mentally fatigued, which might have attenuated the mental fatigue effects. That is, considering the coupling tendencies that emerge between confronting teams, it is likely that the behavior of one team influenced the opposing team to behave in a similar way (Duarte, Araujo, Correia, et al., 2013). In this sense, it is possible that the mental fatigued team might have been constrained to act in a synchronized manner with the opponents (without mental fatigue), and consequently might have decreased the potential effects of mental fatigue. Therefore, additional research is required to better understand the magnitude of the effects of the mental fatigue on players physical and tactical performances.

Overall, research has shown that soccer players' behavior is highly sensitive to environmental, task and individual constraints. In fact, several reports have shown that players' movement behaviors emerge as consequence of changes in task constraints during small-sided games (SSG) (Ade, Harley, \& Bradley, 2014; Aguiar, Gonçalves, Botelho, Lemmink, \& Sampaio, 2015; Gonçalves, Marcelino, Torres-Ronda, Torrents, \& Sampaio, 2016; Hill-Haas, Dawson, Impellizzeri, \& Coutts, 2011; Sampaio, Lago, Gonçalves, Macas, \& Leite, 2014; Travassos et al., 2014). The SSG are modified games, that are usually performed in smaller pitches, involving a smaller number of players and with adapted rules (Hill-Haas et al., 2011). Accordingly, the SSG have been extensively used to manipulate the tasks constraints, while capturing the players physical, technical and tactical performances (Aguiar et al., 2015; Badin 
et al., 2016; Gonçalves, Esteves, et al., 2016; Hill-Haas et al., 2011). The results revealed that by manipulating the task constraints during SSG is it possible to change the game demands with implications in muscular and mental fatigue (Aguiar et al., 2015; Gonçalves, Esteves, et al., 2016; Gonçalves, Marcelino, et al., 2016; Hill-Haas et al., 2011; Travassos et al., 2014). However, little is known of how changes in individual constraints, such as the muscular and mental fatigue, might affect players' physical and tactical performance. Therefore, the aim of this study was to examine the effects of mental and additional muscular fatigue on soccer physical and tactical behavior. It was hypothesized that with additional muscular fatigue, players are likely to demonstrate a more regular movement behavior, while decreasing the physical performance. Additionally, since mental fatigue affects players' opportunities for action, a decrease in distance covered at moderate to high speeds, as well as in the time spent synchronized in the longitudinal direction might be expected to result from mental fatigue.

\section{Methods}

\subsection{Participants}

Ten amateur youth soccer players (age $=13.7 \pm 0.5 \mathrm{y} ;$ height $=163.1 \pm 6.1 \mathrm{~cm}$; body mass $=56.2 \pm 4.2 \mathrm{~kg}$; with $6.1 \pm 0.9$ years of experience; and $5.0 \pm 2.6$ years of playing together; maximum SSG heart rate $198.4 \pm 5.4$ beat $\cdot \mathrm{min}^{-1}$ ) from a regional soccer academy in Portugal participated in this study. The players maturity status was calculated from a predictive equation based on chronological age, standing height, sitting height and body mass, which have been considered as a reliable, non-invasive and practical approach to measure the maturity of youth players (Mirwald, Baxter-Jones, Bailey, \& Beunen, 2002). All players were considered in the normal maturity stages and their mean age of peak height velocity was $-0.04 \pm 0.41$ years (Mirwald et al., 2002), suggesting that each were circum-PHV $(-1$ to +1 years from the PHV), which is the age were maximum rate of growth occurs (Selmi, Al-Haddabi, Yahmed, \& Sassi, 2017). While previous studies have reported differences in physical performance between groups from different maturity stages (Selmi et al., 2017), all players tested were within the circum-PHV group, which may suggest negligible within-group effect of maturation. All players performed 3 training sessions (90-115 min) per week and played an official eleven-a-side game during the weekend at a regional playing standard in a regular football field (104 × $64 \mathrm{~m})$. Two goalkeepers were part of the study, but were excluded from the analysis. An informed and written consent was provided to the coaches, players, and their parents, as well as by the club, before the beginning of the study. All participants were notified that they could withdraw from the study at any time. The study protocol followed the guidelines and was approved by the Local Ethics Committee and conformed to the recommendations of the Declaration of Helsinki

\subsection{Procedures}

Firstly, all participants completed a familiarization session where they experienced all testing procedures, such as the subjective rates (CR10-scale and visual analogue scale), the Stroop color-word task and the SSG used in the testing session. A standardized soccer SSG was used as the main testing task. The head coach selected the ten best players from the team and divided them into two balanced teams according to his perception of their skill levels in ball passing, ball control, shooting and game knowledge (Sampaio, Lago, et al., 2014). Before the testing session, there was a standardized 15-min warm-up based on running and a ball possession game. The SSG were performed under three different initial conditions performed in a random order: (i) normal condition; (ii) after additional muscular fatigue; (iii) after induced mental fatigue.

\subsection{Small-sided games}

Each team played three, 5-a-side SSG (plus goal keepers) on a natural grass $30 \times 25$-m (length $\mathrm{x}$ width) pitch using formal 7-aside goals (Travassos et al., 2014). The present format was used based on the study of Aguiar et al. (2015), which shown that 4 and 5a-side formats were the minimum formats required to analyze the team tactical behavior. Moreover, the 5-a-side format plus goalkeeper was selected on the basis that it provides a more balanced positional distribution on the pitch compared to the 4-a-side plus goalkeeper. The SSG of each condition lasted for a total of 24-min and included 3 bouts of 6 min match play interspersed with a 3-min recovery period. During the experimental conditions, all the players were under the same treatment condition, e.g., both teams were induced with muscular fatigue at the same time, as well as, both performed the mental fatiguing task at the same time. Although all conditions were performed within the same day (see Fig. 1), players were provided a 45-min recovery period between treatments to ensure that performance was not impaired by the previous condition. Although dependent on the exercise intensity and duration, it has been shown that central fatigue may recover after 30-min of the exercise, while the indicators of muscular fatigue seems to return to values similar to the baseline after $\sim 50$-min (Carroll, Taylor, \& Gandevia, 2016). Furthermore, players spent 54-min performing the SSG, which is below than the time of an official match for this age group (70-min). The repeated measures design resulted in 9 different SSG bouts for each participant (i.e. $3 \times 6 \mathrm{~min}$ bouts of SSG for each condition), providing total of 90 samples that were retained for analysis.

\subsection{Treatments}

\subsubsection{Control condition}

In this condition, the players were not under the effect of any of the other initial conditions and started to play the SSG situation 

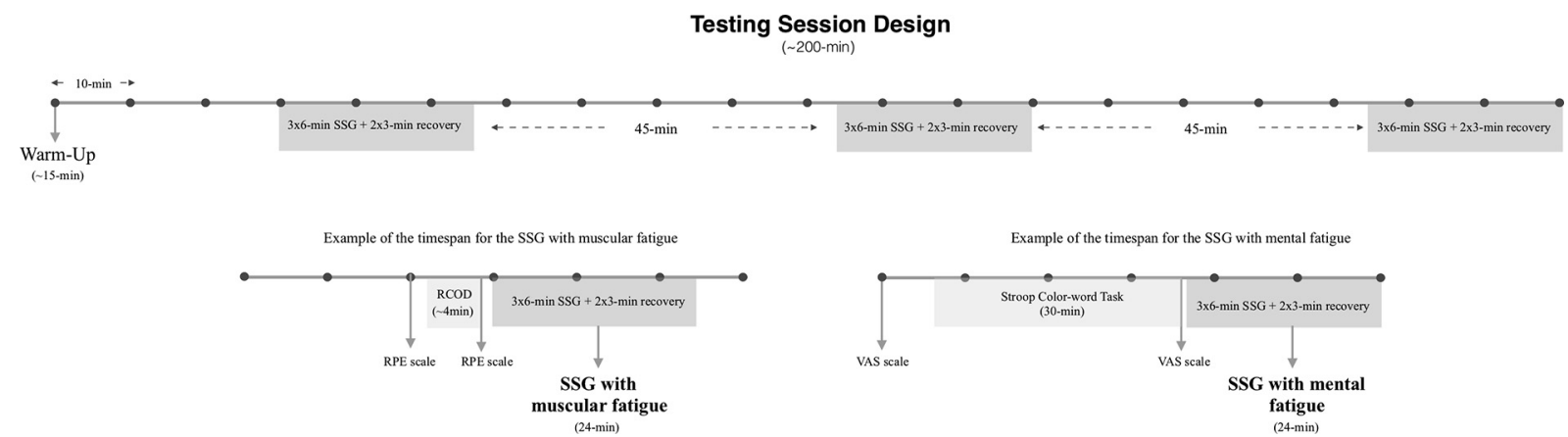

Fig. 1. Data design for the SSG scenarios considering the individual constraint condition. Note: grey dots represent blocks of 60 -min; black dots represent blocks of 10 min; Grey rectangle represent the duration of the SSG conditions. RCOD - repeated change of direction.

after declaring their rate of perceived exertion (RPE) values.

\subsubsection{Muscular fatigue condition}

The additional muscular fatigue was induced with the repeated change of direction (RCOD) task, which was used to add muscular fatigue previous to the SSG. The RCOD protocol (Beckett, Schneiker, Wallman, Dawson, \& Guelfi, 2009) required players to complete $6 \times 20$-m efforts each with four $100^{\circ}$ directional changes every four meters with $25 \mathrm{~s}$ of active recovery between bouts. This task was used based on its potential to induce muscle damage actions, such as accelerations, decelerations and changes of direction (Nedelec et al., 2012). Apparently, the main cause of fatigue during intermittent sprint exercises is the muscular fatigue, as reduced changes in the neural drive have been found (Bishop, 2012; Tomazin et al., 2016). During the RCOD task the players were challenged to perform the task in the minimum time possible during all bouts.

\subsubsection{Mental fatigue condition}

To induce mental fatigue, players performed a 30-min computerized version of Stroop color-word task (Laursen, Jensen, Garde, \& Jorgensen, 2002; Smith, Coutts, et al., 2016). The Stroop-color word task has been extensively used by the available research in sport settings (Badin et al., 2016; Smith, Coutts, et al., 2016; Smith, Zeuwts, et al., 2016), based on its potential to induce mental fatigue (Shou \& Ding, 2013). In this cognitive task, four words (red, blue, green and yellow) were displayed one at a time on a computer screen with a grey background. Players were requested to respond to the selected letters on a computer keyboard by pressing one of four keys that match the color of the word, instead of its meaning. That is, when the word red appeared with the blue color, the correct response was pressing the blue key. However, to increase task difficulty and attention demands, if the color displayed in the screen was red, the correct response was pressing the meaning of the word (e.g., if the word green appears displayed in red, the correct response was press the green key). The task contained 50\% of trials in a congruent fashion (matching word and color) and $50 \%$ in an incongruent way. Each word was presented on the screen during $1000 \mathrm{~ms}(\mathrm{~ms})$, followed by a black screen during another $1000 \mathrm{~ms}$, period after which a new word was displayed. As so, a new word was presented at each $2000 \mathrm{~ms}$, resulting in a total of 900 stimulus during the all test. Incorrect and missing answers ( $>1500 \mathrm{~ms}$ ) induced a beep sound to alert players to be faster or more accurate (Badin et al., 2016). To increase motivation, participants were challenged to complete as fast, successful and accurate as possible more words than the other participants within the 30 -min period.

\subsection{Data collection}

\subsubsection{Subjective ratings psychological states}

The CR10-scale (RPE) was recorded both before and after the completion of the RCOD. A 100-mm visual analogue scale (VAS) was used to assess the players' perceptions of mental fatigue immediately prior to, and after the conclusion of the mental fatigue task, and at the end of the SSG (Lee, Hicks, \& Nino-Murcia, 1991). With the CR10-scale the players were required to point to a number which corresponded to their rating, while with the visual analogue scale, players were instructed to mark a vertical line anywhere along the $100-\mathrm{mm}$ scale that reflected their current state.

\subsubsection{Physical activity and positional data}

Positional data, accelerations and distance covered during SSG were gathered using $5 \mathrm{~Hz}$ global positioning system (GPS) units (SPI-PRO, GPSports, Canberra, ACT, Australia) (Coutts \& Duffield, 2010). The players' latitude and longitude coordinates obtained through GPS units were exported and computed using appropriate routines in Matlab ${ }^{\circledR}$ (MathWorks, Inc., Massachusetts, USA). Additionally, missing data were re-sampled and tracking error noise was reduced by smoothing the data based on a $3 \mathrm{~Hz}$ Butterworth low pass filter (Folgado, Duarte, Fernandes, \& Sampaio, 2014).

The dynamic positional data of players were used to determine the distance between dyads, i.e., pair of players that share the same surrounding and have the intention to achieve the same goal, from the same team (Gonçalves, Esteves, et al., 2016), the teams' stretch index (represented by the mean distances from each player to the team geometrical centers of gravity of the teams) (Travassos 
et al., 2014), and the time that the dyads spent synchronized in both longitudinal and lateral directions, i.e., by sharing the same goal the players from the same team are likely to move horizontally and vertically in the pitch in a coordinated-based trend (Folgado et al., 2014). The time that players spent synchronized in both the longitudinal and lateral movement directions was calculated for all possible dyads taking into consideration the five outfield teammates, based on the Hilbert Transform (Palut \& Zanone, 2005). Also, the regularity in the distance between dyads was assessed using approximate entropy (ApEn). The imputed values used to compute were 2 to vector length (m) and $0.2^{*}$ std to the tolerance (r) (Yentes et al., 2013). The ApEn values range from 0 to 2, and lower values represents more repeatable patterns, i.e., values closer to 0 means that the distance between players are likely to be maintained during the game in a more regular pattern.

Both total distance covered and ratios of distances covered at three speed zones (i.e. high ratio $(>16 \mathrm{~km} / \mathrm{h})$, moderate ratio $(10.0-15.9 \mathrm{~km} / \mathrm{h})$ and lower ratio $(7.0-9.9 \mathrm{~km} / \mathrm{h})$ speeds) were calculated. To assist comparison, the three speed zones were expressed relative to total distance covered and were normalized for each $100 \mathrm{~m}$ (Abade, Gonçalves, Leite, \& Sampaio, 2014). In addition, the number of all accelerations and decelerations were measured for the category $\left(0.5-3.0 \mathrm{~m} / \mathrm{s}^{2}\right.$ ) (Ade et al., 2014).

\subsection{Statistical analysis}

The descriptive statistics were reported as mean and standard deviations for all variables. Comparison between conditions (control vs. muscular fatigue, control vs. mental fatigue, muscular fatigue vs. mental fatigue) were determined using standardized mean differences with 90\% confidence intervals (Cumming, 2012; Hopkins, Marshall, Batterham, \& Hanin, 2009). Effect sizes statistics (Cohen's $d$ ) were assessed using the following ranges: < 0.2, trivial; 0.2-0.59, small; 0.60-1.19, moderate; 1.20-1.99, large; and $>2.0$, very large (Hopkins et al., 2009). Differences in group means were expressed in percentage units with $90 \%$ confidence limits (CL). Smallest worthwhile differences were assessed using the standardized units multiplied by 0.2 . Uncertainty in the true effects of the conditions were evaluated through non-clinical magnitude-based inferences. Magnitudes of clear effects were considered as the following scale: 25-75\%, possibly; 75-95\% likely; 95-99\%, very likely; > 99\% most likely (Hopkins et al., 2009).

\section{Results}

\subsection{Subjective ratings}

The RPE and mental fatigue responses to the muscular and mental induced fatigue conditions are shown in Fig. 2. The RCOD task most likely increased the RPE values when compared the pre-treatment to the post-treatment (very large effect, 2.4 ; $\pm 90 \mathrm{CI}, \pm 0.6$ ). Similarly, the Stroop color-word Task most likely increased the level of mental fatigue between the pre-treatment and the posttreatment (moderate effect, $1.0 ; \pm 0.4)$. During the mental fatigue task, the players provided $\sim 88 \%$ correct answers $(794.8 \pm 56.3$ ), and $\sim 12 \%$ incorrect answers $(105.1 \pm 56.3)$, with an average time of response of $0.75( \pm 0.22) \mathrm{ms}$.

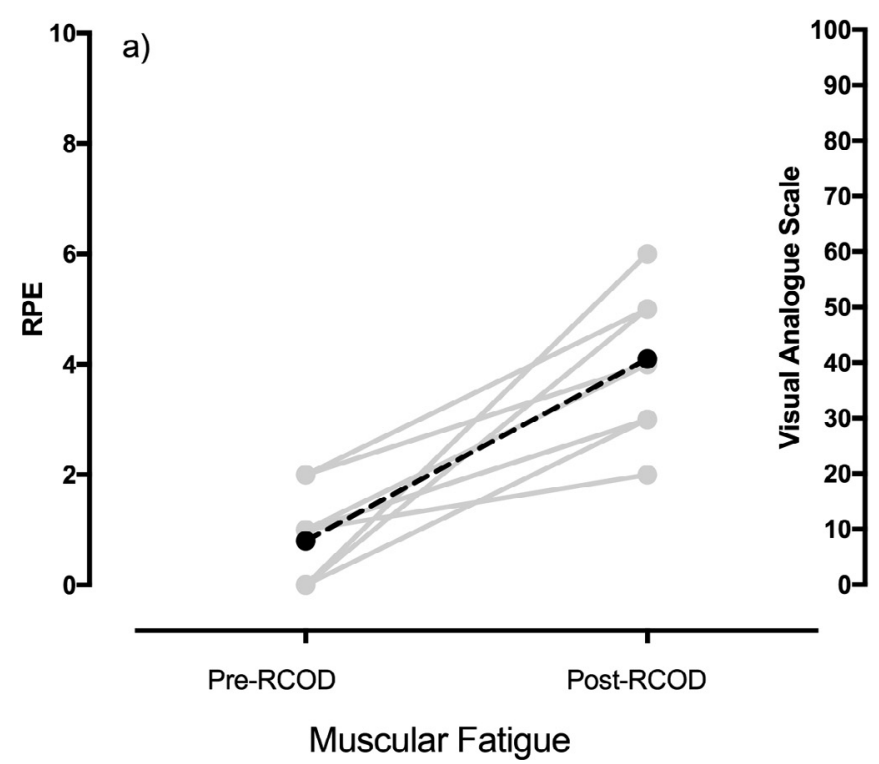

b)

Fig. 2. Effects of muscular fatigue task on RPE and effects of mental fatigue task on visual analogue scale. a) Effects of muscular fatigue task on the levels of RPE; b) Effects of mental fatigue task on visual analogue scale. Note: grey solid lines indicated responses of individual participants; black dotted lines indicated mean value. RPE - rating of perceived exertion. RCOD - repeated change of direction. 

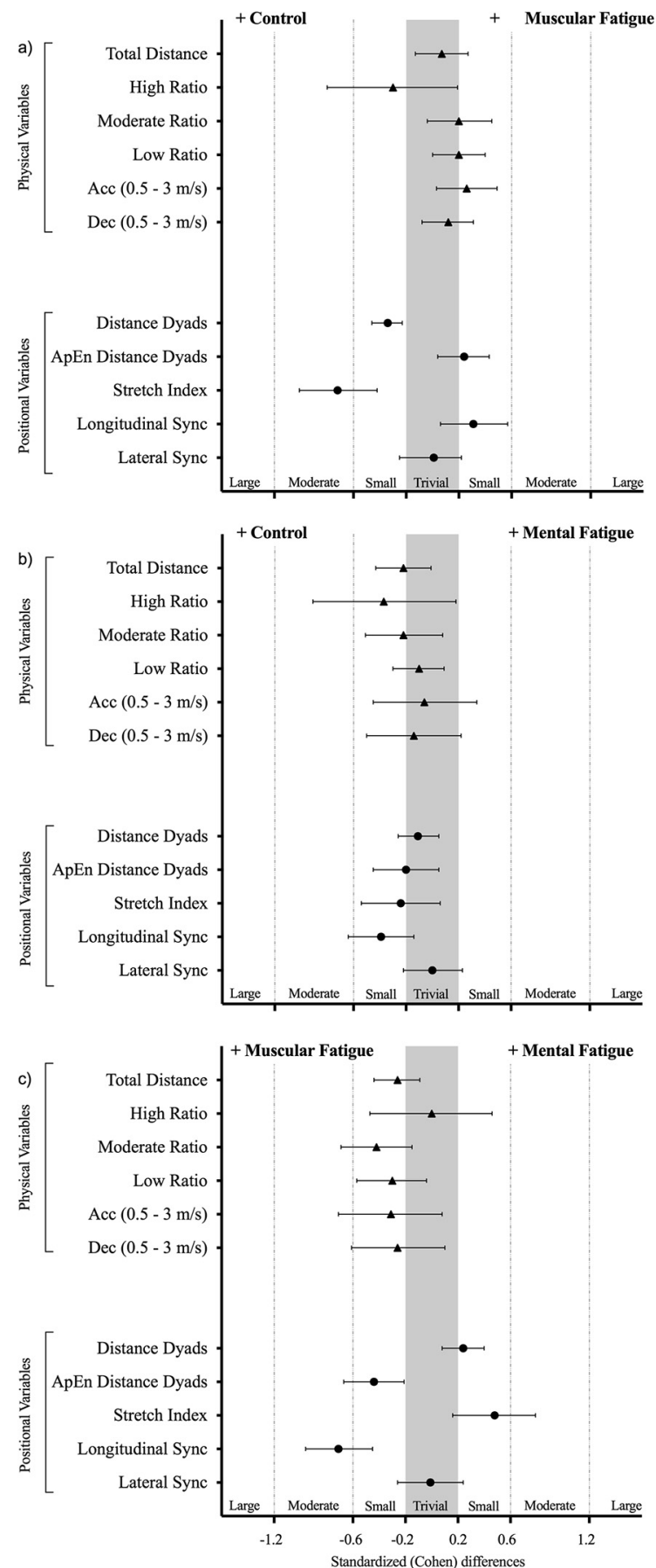

Fig. 3. Standardized (Cohen's $d$ ) differences in muscular and positional variables according to between the treatment conditions (3a. Control vs. Muscular fatigue; 3b. Control vs. Mental Fatigue; 3c. Muscular fatigue vs. Mental Fatigue). Error bars indicate uncertainty in the true mean changes with $90 \%$ confidence intervals. Dec decelerations, Acc - accelerations, ApEn - approximate entropy, Sync - synchronization.

\subsection{Physical performance}

Most of the physical activity variables increased with muscular fatigue compared to the control condition, with the exception of high ratio (see Fig. 3a and Table 1). In contrast, the muscular condition resulted in a possible $\sim 38 \%$ decrease in the ratio of distances covered at high speeds (small effect) compared to the control condition.

Overall, the mental fatigue condition resulted in decreased values in all physical variables than the control condition, mainly in relation to the high and moderate ratio (see Fig. $3 \mathrm{~b}$ and Table 1 ). With mental fatigue, there was a likely $\sim 3 \%$ decrease in total 
Table 1

Descriptive analysis for both positional and physical variables when varying the initial condition.

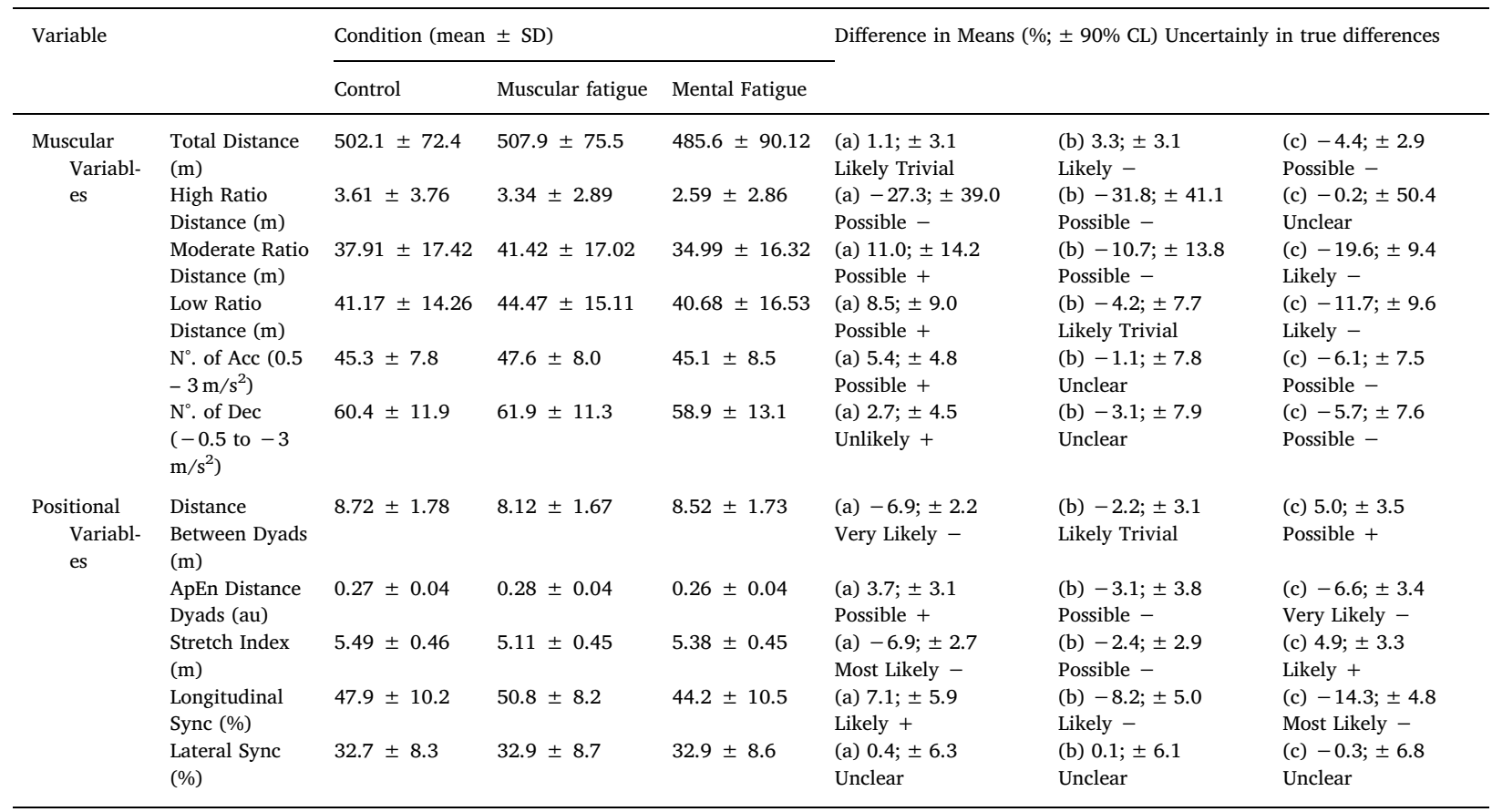

Note: ApEn = approximate entropy; Dec = Deceleration; Acc = Acceleration; CL = confidence limits; $+=$ increase; $-=$ decrease. Differences in means $(\% ; \pm 90 \%$ $\mathrm{CL}$ ) are presented as (a) Control condition vs muscular fatigue condition; (b) Control condition vs mental fatigue condition; (c) Muscular fatigue condition vs mental fatigue condition.

distance covered and a possible $\sim 11 \%$ decrease in the moderate ratio (moderate effects), and a possible $\sim 32 \%$ decrease in the ratio of distances travelled at high speeds (small effects, respectively) compared to the control condition.

The muscular fatigue condition increased physical activity variables compared to the mental fatigue condition (see Fig. 3c and Table 1). The muscular fatigue condition resulted in a possible $\sim 4 \%$ increase in total distance covered, likely $\sim 20 \%$ increase in the moderate ratio, and a possible $\sim 6 \%$ increase in the number of accelerations $\left(0.5-3.0 \mathrm{~m} / \mathrm{s}^{2}\right.$ ) (small effects, respectively).

\subsection{Tactical behavior}

There was a very likely $\sim 6.9 \%$ decrease in the distance between dyads (small effect) and a possible $\sim 3 \%$ increase on the ApEn distance dyads (i.e., a decrease in the regularity of the distance between dyads) (small effect) with muscular fatigue compared to the control condition (see Fig. 3a and Table 1). There was also a likely $\sim 7 \%$ decrease in the team's stretch index with muscular fatigue compared to the control condition (moderate effect). Additionally, the muscular fatigue condition also most likely decreased ( $\sim 7 \%$ ) the time spent synchronized in longitudinal displacements compared to the control condition (small effect).

The mental fatigue condition resulted in a decrease in most of the tactical variables compared to the control condition (see Fig. $3 \mathrm{~b}$ and Table 1). There was also a possible $\sim 2 \%$ decrease in the team stretch index (small effect) and a likely $\sim 8 \%$ decrease in the time that players spent synchronized in the longitudinal displacements (small effect) than in the control condition. Although a likely trivial decrease was observed in the distance between dyads between the mental fatigue and control conditions, the mental fatigue condition showed a $\sim 3 \%$ increase in the regularity between the dyads distance (small effect) compared to the control condition.

The muscular fatigue condition resulted in higher ApEn distance dyads and longitudinal synchrony than the mental fatigue condition (see Fig. 3c and Table 1). There was a very likely $\sim 7 \%$ increase in the regularity in the distance between dyads (small effect) and a most likely $\sim 14 \%$ increase in the time that players spent synchronized in longitudinal displacements (moderate effect) during muscular fatigue condition compared to the mental fatigue condition. In contrast, the mental fatigue condition showed a possible $5 \%$ increase in the distance between dyads (small effect), and a likely $\sim 5 \%$ increase in the team stretch index (small effect) than with muscular fatigue.

\section{Discussion}

The aim of the present study was to examine the effects of induced mental and muscular fatigue on physical activity and tactical performance measures during soccer SSG. The results indicated a general effect of muscular and mental fatigue on players' physical performance during soccer SSG. Specifically, muscular fatigue decreased distance covered at higher speeds, however it also increased 
the moderate and low ratio, as well as the number of accelerations. In turn, mental fatigue showed lowest values in most of the physical variables. From the tactical perspective, the muscular fatigue decreased the regularity on distance between dyads, and increased the time spent synchronized in longitudinal displacements compared to the mental fatigue.

In relation to the control condition, the muscular fatigue condition decreased the distance between dyads. This decrease may have result as a self-organizing behavior to reduce the need to adjust their positioning during specific moments, such as moments of ball loss, and consequently decreasing the need to perform high-intensity actions. These findings demonstrate that with increased muscular fatigue, players are likely to reduce the distance between their teammates resulting in decreased player dispersion (i.e. reduced stretch index) as a preferable movement behavior. This may emerge as a collective strategy to overcome the effects of muscular fatigue, allowing the players to adopt more stable behaviors and of less risk under fatigue conditions (Duarte, Araujo, Folgado, et al., 2013). Other studies have shown that players adopt similar collective strategies when they were under the effect of specific constraints, such as playing in numerical inferiority (Sampaio, Lago, et al., 2014). Moreover, the increased positional regularity to the team centroid suggested that players possibly adopt more pre-structured strategical behaviors (Sampaio, Lago, et al., 2014). In this sense, it is possible that players look for more stable movement behaviors, such as decreasing the distance between teammates, when they perceive that the task and/or individual constraints were somehow affected.

The players' positioning and movements are result from the interaction between the environment information and players actions, and thus, a better knowledge of players performance may be considered if the tactical and physical performances are analyzed together (Folgado, Duarte, Marques, \& Sampaio, 2015; Gonçalves, Esteves, et al., 2016). Accordingly, the results also revealed a decrease in the distance covered in the high ratio, which seems to be linked with the decreased distance between dyads. In fact, it has previously been established that soccer players adjust their speed movements according to the environment, the ball, teammates and opponents (Travassos et al., 2014). Thus, it is likely that the players' ability to undertake these high intensity efforts were constraint by the interpersonal distance between players. While there was found a decrease in the high ratio, in turn there was also an increase in the moderate and low ratios, which can be linked with the need of players to move to maintain the team synchrony (Gonçalves, Esteves, et al., 2016). That is, players may have to cover more distance in low to moderate speeds to compensate the lack of team dispersion. In fact, the higher number of accelerations $(0.5-3 \mathrm{~m} / \mathrm{s})$ found in the physical fatigue condition in relation to the control condition may reinforce this finding, as players may have to accelerate more often to obey the soccer game principles, such as delay or mobility, due to their lower dispersion on pitch.

The level of team synchrony has also been shown to be dependent upon the spatial proximity between players, with player's movements tending to be more tightly coupled with the nearest neighboring players (Folgado et al., 2014). Accordingly, the lower team dispersion with increased muscular fatigue compared to the control condition may help to explain the increase in the longitudinal teams' synchronization. Moreover, soccer players have been shown to be more synchronized in longitudinal movements during low and very high-speed activities (Folgado et al., 2014). Consequently, it is possible that the increased longitudinal coordination with muscular fatigue was linked with the decrease in the speed of the SSG, which is supported by the lower distances covered at higher speeds $(-27.3 \%)$ during the muscular condition.

In the present investigation, the physical activity profile during the SSG were lowest following mental fatigue condition. These results are contradictory to those found in previous reports that analyzed the player's physical performance when mentally fatigued during SSG (Badin et al., 2016; Coutinho et al., 2017). While in this study both confronting teams were mentally fatigued, in turn, in these previous studies there was only one team mentally fatigued. Accordingly, player's movement behavior seems to be influenced by contextual and tactical factors (Badin et al., 2016). In fact, since opposing teams have shown coupling tendencies (Duarte, Araujo, Folgado, et al., 2013; Gonçalves, Esteves, et al., 2016), it is possible that the behavior of one team (without mental fatigue), attracted the opposite team (with mental fatigued) to behave in a similar fashion, resulting in identical physical performance. In contrast, in this study both teams were mentally fatigued, which may have limited the tolerance for the exercise (Marcora, Staiano, \& Manning, 2009). As consequence, the players may have decreased their physical performance to be able to cope with the task until the end (Smith et al., 2015).

A new finding from the present study was the effects of mental fatigue on tactical behavior taking into consideration that both teams were under the same effect, with the main results revealing that there were more regular distances between dyads and a decrease in team dispersion with mental fatigue compared to the control condition. Additionally, despite having greater within-team player density and with more regular distances between teammates, the players were less coupled in longitudinal displacements than the other experimental conditions. These results suggest that mental fatigue affects the manner in which players perceive and explore possibilities for action during SSG, with a clear reduced movement synchronization between teammates (Coutinho et al., 2017). Our results are consistent with the results found in both laboratory studies and game-based situations. For instance, laboratory-based studies shown that under mental fatigue there is a decrease in the ability to perceive the appropriate environmental information (Boksem et al., 2006), as well as to properly monitor and adjust the performance (Lorist et al., 2005), such as the players positioning. As consequence of a reduced ability to interact with the environment when mentally fatigued, players may make decisions about their positioning using inappropriate information (Coutinho et al., 2017; Lorist et al., 2005) and this may result in players failing to properly identify and use the available pitch space. Similarly, previous studies showed that mental fatigue decreases in the time spent synchronized in the lateral direction, and additional spatial references (i.e. line markings) increased time spent in the longitudinal direction during SSGs (Coutinho et al., 2017). Together, these previous results suggest that mental fatigue may cause players to fail to notice important information in a timely manner and this may result in altered positioning between teammates and decreasing the level of movement synchronization.

Interestingly, ApEn values revealed higher movement irregularity following the muscular fatigue condition than in both the mental fatigue of control condition. Nevertheless, players were more synchronized during following muscular fatigue, and the 
distances between teammates were more variable than both mental fatigue and control conditions. These observations show that despite being able to vary distances between players - likely due to game demands and players' capabilities - players were able to maintain the synchrony with their teammates. In contrast, mental fatigue resulted in greater stability of the distances between players within the same team but with lower levels of between-player synchrony. A possible explanation for these observations is that mental fatigue impairs players' perception of available information and also might affect their ability to identify suitable possibilities for action (Boksem et al., 2006; Coutinho et al., 2017; Nedelec et al., 2012; Sampaio, Gonçalves, et al., 2014; Smith, Zeuwts, et al., 2016).

Whilst the present study induced muscular and mental fatigue using protocols that are not common in soccer, the findings do have important practical applications that may be used to alter tactical behavior. Applying a fatigue task prior to game-based situations may be a useful solution to promote compact behaviors and movement synchronization, while increasing the irregularity in the distance between players. However, it is important for coaches to know that despite the decrease in the distance covered at high intensities due to the lower team dispersion, it may also increase the need to cover more distance at low to moderate speeds and perform more accelerations to maintain the team synchrony. In contrast, coaches should also be aware that mental fatigue is likely to decrease synchronization in goal-to-goal displacements and activity profile and, thus, coaches should carefully control the use of mental tasks near to the match-day. Moreover, while a previous study used an ecological task to induce mental fatigue (Coutinho et al., 2017), the type of task used might increase the physical training stimulus to the players, and therefore the Stroop-color word task may emerge as an suitable alternative to induce mental fatigue in a more controlled environment.

Although this study adds to the understanding of physical and tactical performance under muscular and mental fatigue there are some limitations that should be acknowledged. In the present study, the players reported higher mental fatigue following the mental fatigue task, suggesting that the Stroop-color word task was effective in inducing mental fatigue. However, other markers should be used in complement to confirm this assessment, such as the frontal theta waves which can be assessed with an electroencephalogram test (Wascher et al., 2014). The level of motivation of the players could also have been monitored, as it has been shown that motivation may impact more the performance than the mental fatigue in longer lasting exercise protocols (Mockel, Beste, \& Wascher, 2015; Wascher et al., 2014). It is possible that the performance measures in the present study might have been influenced by reduced player motivation in the final test conditions. Another possible limitation of the present study was that the same muscular and mental fatigue protocol to all subjects. Individualized fatigue protocols may be required according to the individual threshold of the players should be investigated in future studies. Finally, this study used three experimental conditions to investigate fatigue (control, muscular fatigue and mental fatigue) and these were completed during one session in a random order. Accordingly, future studies may investigate the effects of predetermined order conditions on players' performance. Moreover, different effects of muscular and mental fatigue might emerge when the number of players are manipulated, and so, future studies should also inspect how players movement behavior is affected by different number of players (e.g., 3vs3, 6vs6, 9vs9).

\section{Conclusions}

This study demonstrated that both muscular and mental fatigue can modify players' physical and tactical performances. After inducing muscular fatigue, there was an increase in longitudinal synchronization, possibly as result of the higher spatial proximity. The lower team dispersion seems to require the players to cover more distance at low to moderate speeds and to perform more accelerations to compensate the higher compactness with muscular fatigue. Following the mental fatigue task, we observed a decrease in longitudinal synchronization, even with the decrease in the team stretch index and with the higher regularity in the distances between players. These results may suggest that players' ability to use the environmental information to support their actions may be impaired when mentally fatigued, which may affect positioning on the pitch. The lower distance covered at moderate to high speeds, indicates that high-intensity exercise is impaired when players are mentally fatigued. Overall, coaches may use both situations during training to prepare the players to deal with these scenarios on the performance contexts. Additionally, coaches should be aware that that the training session design that changes level of player muscular and mental fatigue may influence changes in players' movement behavior. Further manipulations should be tested with variation on different levels of players' fatigue and also different levels of players' expertise.

\section{Acknowledgments}

This work was supported by the Portuguese Foundation for Science and Technology (FCT, Portugal) through a Doctoral grant endorsed to the first author (SFRH/BD/105081/2014) under the Human Potential Operating Program (POPH). Research Project NanoSTIMA: Macro-to-Nano Human Sensing: Towards Integrated Multimodal Health Monitoring and Analytics, NORTE-01-0145FEDER-000016, Fundo Europeu de Desenvolvimento Regional (FEDER) - NORTE 2020.

\section{Appendix A. Supplementary data}

Supplementary data associated with this article can be found, in the online version, at http://dx.doi.org/10.1016/j.humov.2018. 03.004.

\section{References}


under-19 elite Portuguese players. International Journal of Sports Physiology and Performance, 9, 463-470.

Ade, J. D., Harley, J. A., \& Bradley, P. S. (2014). Physiological response, time-motion characteristics, and reproducibility of various speed-endurance drills in elite youth soccer players: Small-sided games versus generic running. International Journal of Sports Physiology and Performance, 9, 471-479.

Aguiar, M., Gonçalves, B., Botelho, G., Lemmink, K., \& Sampaio, J. (2015). Footballers' movement behaviour during 2-, 3-, 4- and 5-a-side small-sided games. Journal of Sports Sciences, 33, 1259-1266.

Badin, O. O., Smith, M. R., Conte, D., \& Coutts, A. J. (2016). Mental fatigue: Impairment of technical performance in small-sided Soccer games. International Journal of Sports Physiology and Performance, 11, 1100-1105.

Beckett, J. R., Schneiker, K. T., Wallman, K. E., Dawson, B. T., \& Guelfi, K. J. (2009). Effects of static stretching on repeated sprint and change of direction performance. Medicine and Science in Sports and Exercise, 41, 444-450.

Bishop, D. J. (2012). Fatigue during intermittent-sprint exercise. Clinical and Experimental Pharmacology and Physiology, 39, 836-841.

Boksem, M. A., Meijman, T. F., \& Lorist, M. M. (2006). Mental fatigue, motivation and action monitoring. Biological Psychology, 72, $123-132$.

Bradley, P. S., Sheldon, W., Wooster, B., Olsen, P., Boanas, P., \& Krustrup, P. (2009). High-intensity running in English FA Premier League soccer matches. Journal of Sports Sciences, 27, 159-168.

Bush, M., Barnes, C., Archer, D. T., Hogg, B., \& Bradley, P. S. (2015). Evolution of match performance parameters for various playing positions in the English Premier League. Human Movement Science, 39, 1-11.

Carroll, T. J., Taylor, J. L., \& Gandevia, S. C. (2016). Recovery of central and peripheral neuromuscular fatigue after exercise. Journal of Applied Physiology (1985).

Castellano, J., Blanco-Villaseñor, A., \& Alvarez, D. (2011). Contextual variables and time-motion analysis in soccer. Journal of Sports Science and Medicine, 32, 415-421.

Coutinho, D., Gonçalves, B., Travassos, B., Wong, D. P., Coutts, A. J., \& Sampaio, J. E. (2017). Mental fatigue and spatial references impair soccer players' physical and tactical performances. Frontiers in Psychology, 8, 1645.

Coutts, A. J., \& Duffield, R. (2010). Validity and reliability of GPS devices for measuring movement demands of team sports. Journal of Science and Medicine in Sport, 13, 133-135.

Cumming, G. (2012). Understanding the new statistics: Effect sizes, confidence intervals, and meta-analysis. Routledge: Taylor \& Francis Group.

Duarte, R., Araujo, D., Correia, V., Davids, K., Marques, P., \& Richardson, M. J. (2013). Competing together: Assessing the dynamics of team-team and player-team synchrony in professional association football. Human Movement Science, 32, 555-566.

Duarte, R., Araujo, D., Folgado, H., Esteves, P., Marques, P., \& Davids, K. (2013). Capturing complex, non-linear team behaviours during competitive football performance. Journal of Systems Science and Complexity, 26, 62-72.

Folgado, H., Duarte, R., Fernandes, O., \& Sampaio, J. (2014). Competing with lower level opponents decreases intra-team movement synchronization and time-motion demands during pre-season soccer matches. PLoS One, 9, e97145.

Folgado, H., Duarte, R., Marques, P., \& Sampaio, J. (2015). The effects of congested fixtures period on tactical and physical performance in elite football. Journal of Sports Sciences, $33,1238-1247$.

Gandevia, S. C. (2001). Spinal and supraspinal factors in human muscle fatigue. Physiological Reviews, 81, 1725-1789.

Gonçalves, B., Esteves, P., Folgado, H., Ric, A., Torrents, C., \& Sampaio, J. (2016). Effects of pitch area-restrictions on tactical behavior, physical and physiological performances in soccer large-sided games. Journal of Strength \& Conditioning Research Advance Online Publication.

Gonçalves, B., Marcelino, R., Torres-Ronda, L., Torrents, C., \& Sampaio, J. (2016). Effects of emphasising opposition and cooperation on collective movement behaviour during football small-sided games. Journal of Sports Sciences, 34, 1346-1354.

Hill-Haas, S. V., Dawson, B., Impellizzeri, F. M., \& Coutts, A. J. (2011). Physiology of small-sided games training in football: A systematic review. Sports Medicine, 41, 199-220.

Hopkins, W. G., Marshall, S. W., Batterham, A. M., \& Hanin, J. (2009). Progressive statistics for studies in sports medicine and exercise science. Medicine \& Science in Sports \& Exercise, 41, 3-13.

Knicker, A. J., Renshaw, I., Oldham, A. R., \& Cairns, S. P. (2011). Interactive processes link the multiple symptoms of fatigue in sport competition. Sports Medicine, 41, 307-328.

Laursen, B., Jensen, B. R., Garde, A. H., \& Jorgensen, A. H. (2002). Effect of mental and physical demands on muscular activity during the use of a computer mouse and a keyboard. Scandinavian Journal of Work, Environment \& Health, 28, 215-221.

Lee, K. A., Hicks, G., \& Nino-Murcia, G. (1991). Validity and reliability of a scale to assess fatigue. Psychiatry Research, 36, 291-298.

Lorist, M. M., Boksem, M. A., \& Ridderinkhof, K. R. (2005). Impaired cognitive control and reduced cingulate activity during mental fatigue. Brain Research. Cognitive Brain Research, 24, 199-205.

Marcora, S. M., Staiano, W., \& Manning, V. (2009). Mental fatigue impairs physical performance in humans. Journal of Applied Physiology, $106,857-864$.

Mehta, R. K., \& Agnew, M. J. (2012). Influence of mental workload on muscle endurance, fatigue, and recovery during intermittent static work. European Journal of Applied Physiology, 112, 2891-2902.

Mirwald, R. L., Baxter-Jones, A. D., Bailey, D. A., \& Beunen, G. P. (2002). An assessment of maturity from anthropometric measurements. Medicine \& Science in Sports \& Exercise, 34, 689-694.

Mockel, T., Beste, C., \& Wascher, E. (2015). The effects of time on task in response selection-An ERP study of mental fatigue. Scientific Reports, 5, 10113.

Nedelec, M., McCall, A., Carling, C., Legall, F., Berthoin, S., \& Dupont, G. (2012). Recovery in soccer: Part I- Post-match fatigue and time course of recovery. Sports Medicine, 42, 997-1015.

Newell, K. M. (1986). Constraints on the development of coordination. Springer pp. 341-360.

Palut, Y., \& Zanone, P. G. (2005). A dynamical analysis of tennis: Concepts and data. Journal of Sports Sciences, 23, 1021-1032.

Rampinini, E., Bosio, A., Ferraresi, I., Petruolo, A., Morelli, A., \& Sassi, A. (2011). Match-related fatigue in soccer players. Medicine and Science in Sports and Exercise, 43, 2161-2170.

Sampaio, J., Gonçalves, B., Rentero, L., Abrantes, C., \& Leite, N. (2014). Exploring how basketball players tactical performances can be affected by activity workload. Science \& Sports, 29.

Sampaio, J., Lago, C., Gonçalves, B., Macas, V. M., \& Leite, N. (2014). Effects of pacing, status and unbalance in time motion variables, heart rate and tactical behaviour when playing 5-a-side football small-sided games. Journal of Sports Science and Medicine, 17, 229-233.

Selmi, M. A., Al-Haddabi, B., Yahmed, M. H., \& Sassi, R. H. (2017). Does maturity status affect the relationship between anaerobic speed reserve and multiple sprints sets performance in young soccer players? Journal of Strength \& Conditioning Research.

Shou, G., \& Ding, L. (2013). Ongoing EEG oscillatory dynamics suggesting evolution of mental fatigue in a color-word matching stroop task. In 6th Annual International IEEE EMBS Conference on Neural Engineering. San Diego, California.

Silva, J. R., Rumpf, M. C., Hertzog, M., Castagna, C., Farooq, A., Girard, O., \& Hader, K. (2017). Acute and residual soccer match-related fatigue: A systematic review and metaanalysis. Sports Medicine.

Smith, M. R., Coutts, A. J., Merlini, M., Deprez, D., Lenoir, M., \& Marcora, S. M. (2016). Mental fatigue impairs soccer-specific physical and technical performance. Medicine and Science in Sports and Exercise, 48, 267-276.

Smith, M. R., Marcora, S. M., \& Coutts, A. J. (2015). Mental fatigue impairs intermittent running performance. Medicine and Science in Sports \& Exercise, 47, 1682-1690.

Smith, M. R., Zeuwts, L., Lenoir, M., Hens, N., De Jong, L. M., \& Coutts, A. J. (2016). Mental fatigue impairs soccer-specific decision-making skill. Journal of Sports Sciences, 34, 1297-1304.

Smits, B. L., Pepping, G.-J. J., \& Hettinga, F. J. (2014). Pacing and decision making in sport and exercise: The roles of perception and action in the regulation of exercise intensity. Sports Medicine, 44, 763-775.

Tomazin, K., Morin, J. B., \& Millet, G. Y. (2016). Neuromuscular fatigue aetiology after repeated sprints depends on exercise modality. International Journal of Sports Physiology and Performance, 1-28.

Travassos, B., Gonçalves, B., Marcelino, R., Monteiro, R., \& Sampaio, J. (2014). How perceiving additional targets modifies teams' tactical behavior during football small-sided games. Human Movement Science, 38, 241-250.

Van Cutsem, J., Marcora, S., De Pauw, K., Bailey, S., Meeusen, R., \& Roelands, B. (2017). The effects of mental fatigue on physical performance: A systematic review. Sports Medicine.

Varley, M. C., Elias, G. P., \& Aughey, R. J. (2012). Current match-analysis techniques' underestimation of intense periods of high-velocity running. International Journal of Sports Physiology and Performance, 7, 183-185.

Wascher, E., Rasch, B., Sanger, J., Hoffmann, S., Schneider, D., Rinkenauer, G., ... Gutberlet, I. (2014). Frontal theta activity reflects distinct aspects of mental fatigue. Biological Psychology, 96, 57-65.

Yentes, J. M., Hunt, N., Schmid, K. K., Kaipust, J. P., McGrath, D., \& Stergiou, N. (2013). The appropriate use of approximate entropy and sample entropy with short data sets. Annals of Biomedical Engineering, 41, 349-365. 